\title{
Fine Spectra of Upper Triangular Triple-Band Matrices over the Sequence Space $\ell_{p}(0<p<\infty)$
}

\author{
Ali Karaisa ${ }^{1}$ and Feyzi Başar ${ }^{2}$ \\ ${ }^{1}$ Department of Mathematics, Necmettin Erbakan University, Karaciğan Mahallesi, Ankara Caddesi 74, 42060 Konya, Turkey \\ ${ }^{2}$ Department of Mathematics, Fatih University, Hadımköy Campus, Büyükçekmece, 34500 Istanbul, Turkey
}

Correspondence should be addressed to Ali Karaisa; alikaraisa@hotmail.com

Received 27 September 2012; Revised 28 December 2012; Accepted 31 December 2012

Academic Editor: Simeon Reich

Copyright (C) 2013 A. Karaisa and F. Başar. This is an open access article distributed under the Creative Commons Attribution License, which permits unrestricted use, distribution, and reproduction in any medium, provided the original work is properly cited.

\begin{abstract}
The fine spectra of lower triangular triple-band matrices have been examined by several authors (e.g., Akhmedov (2006), Başar (2007), and Furken et al. (2010)). Here we determine the fine spectra of upper triangular triple-band matrices over the sequence space $\ell_{p}$. The operator $A(r, s, t)$ on sequence space on $\ell_{p}$ is defined by $A(r, s, t) x=\left(r x_{k}+s x_{k+1}+t x_{k+2}\right)_{k=0}^{\infty}$, where $x=\left(x_{k}\right) \in \ell_{p}$, with $0<p<\infty$. In this paper we have obtained the results on the spectrum and point spectrum for the operator $A(r, s, t)$ on the sequence space $\ell_{p}$. Further, the results on continuous spectrum, residual spectrum, and fine spectrum of the operator $A(r, s, t)$ on the sequence space $\ell_{p}$ are also derived. Additionally, we give the approximate point spectrum, defect spectrum, and compression spectrum of the matrix operator $A(r, s, t)$ over the space $\ell_{p}$ and we give some applications.
\end{abstract}

\section{Introduction}

In functional analysis, the spectrum of an operator generalizes the notion of eigenvalues for matrices. The spectrum of an operator over a Banach space is partitioned into three parts, which are the point spectrum, the continuous spectrum, and the residual spectrum. The calculation of these three parts of the spectrum of an operator is called calculating the fine spectrum of the operator.

Over the years and different names the spectrum and fine spectra of linear operators defined by some triangle matrices over certain sequence spaces were studied.

By $\omega$ we denote the space of all complex-valued sequences. Any vector subspace of $\omega$ is called a sequence space. We write $\ell_{\infty}, c, c_{0}$, and $b v$ for the spaces of all bounded, convergent, null, and bounded variation sequences, respectively, which are the Banach spaces with the sup-norm $\|x\|_{\infty}=$ $\sup _{k \in \mathbb{N}}\left|x_{k}\right|$ and $\|x\|_{b v}=\sum_{k=0}^{\infty}\left|x_{k}-x_{k+1}\right|$, respectively, where $\mathbb{N}=\{0,1,2, \ldots\}$. Also by $\ell_{1}$ and $\ell_{p}$ we denote the spaces of all absolutely summable and $p$-absolutely summable sequences, which are the Banach spaces with the norm $\|x\|_{p}=$ $\left(\sum_{k=0}^{\infty}\left|x_{k}\right|^{p}\right)^{1 / p}$, respectively, where $1 \leqslant p<\infty$.
Several authors studied the spectrum and fine spectrum of linear operators defined by some triangle matrices over some sequence spaces. We introduce knowledge in the existing literature concerning the spectrum and the fine spectrum. Cesàro operator of order one on the sequence space $\ell_{p}$ was studied by Gonzàlez [1], where $1<p<\infty$. Also, weighted mean matrices of operators on $\ell_{p}$ have been investigated by Cartlidge [2]. The spectrum of the Cesàro operator of order one on the sequence spaces $b v_{0}$ and $b v$ were investigated by Okutoyi $[3,4]$. The spectrum and fine spectrum of the Rally operators on the sequence space $\ell_{p}$ were examined by Yildırım [5]. The fine spectrum of the difference operator $\Delta$ over the sequence spaces $c_{0}$ and $c$ was studied by Altay and Başar [6]. The same authors also worked out the fine spectrum of the generalized difference operator $B(r, s)$ over $c_{0}$ and $c$, in [7]. Recently, the fine spectra of the difference operator $\Delta$ over the sequence spaces $c_{0}$ and $c$ have been studied by Akhmedov and Başar $[8,9]$, where $b v_{p}$ is the space consisting of the sequences $x=\left(x_{k}\right)$ such that $x=\left(x_{k}-\right.$ $\left.x_{k-1}\right) \in \ell_{p}$ and introduced by Başar and Altay [10] with $1 \leqslant$ $p \leqslant \infty$. In the recent paper, Furkan et al. [11] have studied the fine spectrum of $B(r, s, t)$ over the sequence spaces $\ell_{p}$ and $b v_{p}$ 
with $1<p<\infty$, where $B(r, s, t)$ is a lower triangular tripleband matrix. Later, Karakaya and Altun have determined the fine spectra of upper triangular double-band matrices over the sequence spaces $\mathcal{c}_{0}$ and $c$, in [12]. Quite recently, Karaisa [13] has determined the fine spectrum of the generalized difference operator $A(\widetilde{r}, \widetilde{s})$, defined as an upper triangular double-band matrix with the convergent sequences $\widetilde{r}=\left(r_{k}\right)$ and $\widetilde{s}=\left(s_{k}\right)$ having certain properties, over the sequence space $\ell_{p}$, where $1<p<\infty$.

In this paper, we study the fine spectrum of the generalized difference operator $A(r, s, t)$ defined by a triple sequential band matrix acting on the sequence space $\ell_{p}(0<p<$ $\infty$ ), with respect to Goldberg's classification. Additionally, we give the approximate point spectrum and defect spectrum and give some applications.

\section{Preliminaries, Background, and Notation}

Let $X$ and $Y$ be two Banach spaces and $T: X \rightarrow Y$ be a bounded linear operator. By $R(T)$ we denote range of $T$, that is,

$$
R(T)=\{y \in Y: y=T x, x \in X\} .
$$

By $B(X)$ we also denote the set of all bounded linear operators on $X$ into itself. If $T \in B(X)$ then the adjoint $T^{*}$ of $T$ is a bounded linear operator on the dual $X^{*}$ of $X$ defined by $\left(T^{*} f\right)(x)=f(T x)$ for all $f \in X^{*}$ and $x \in X$.

Let $X \neq\{\theta\}$ be a complex normed space and $T: D(T) \rightarrow$ $X$ be a linear operator with domain $D(T) \subseteq X$. With $T$ we associate the operator $T_{\alpha}=T-\alpha I$, where $\alpha$ is a complex number and $I$ is the identity operator on $D(T)$. If $T_{\alpha}$ has an inverse which is linear, we denote it by $T_{\alpha}^{-1}$, that is,

$$
T_{\alpha}^{-1}=(T-\alpha I)^{-1}
$$

and call it the resolvent operator of $T$.

Many properties of $T_{\alpha}$ and $T_{\alpha}^{-1}$ depend on $\alpha$, and spectral theory is concerned with those properties. For instance, we shall be interested in the set of all $\alpha$ in the complex plane such that $T_{\alpha}^{-1}$ exists. The boundedness of $T_{\alpha}^{-1}$ is another property that will be essential. We shall also ask for what $\alpha$ the domain of $T_{\alpha}^{-1}$ is dense in $X$, to name just a few aspects For our investigation of $T, T_{\alpha}$, and $T_{\alpha}^{-1}$, we need some basic concepts in spectral theory which are given as follows (see [14, pp. 370371]).

Let $X \neq\{\theta\}$ be a complex normed space and $T: D(T) \rightarrow$ $X$ be a linear operator with domain $D(T) \subseteq X$. A regular value $\alpha$ of $T$ is a complex number such that
(R1) $T_{\alpha}^{-1}$ exists,
(R2) $T_{\alpha}^{-1}$ is bounded,
(R3) $T_{\alpha}^{-1}$ is defined on a set which is dense in $X$.

The resolvent set $\rho(T)$ of $T$ is the set of all regular values $\alpha$ of $T$. Its complement $\mathbb{C} \backslash \rho(T)$ in the complex plane $\mathbb{C}$ is called the spectrum of $T$. Furthermore, the spectrum $\sigma(T)$ is partitioned into three disjoint sets as follows. The point spectrum $\sigma_{p}(T)$ is the set such that $T_{\alpha}^{-1}$ does not exist. $\alpha \in \sigma_{p}(T)$ is called an eigenvalue of $T$. The continuous spectrum $\sigma_{c}(T)$ is the set such that $T_{\alpha}^{-1}$ exists and satisfies (R3) but not (R2). The residual spectrum $\sigma_{r}(T)$ is the set such that $T_{\alpha}^{-1}$ exists but does not satisfy (R3).

In this section, following Appell et al. [15], we define the three more subdivisions of the spectrum called the approximate point spectrum, defect spectrum, and compression spectrum.

Given a bounded linear operator $T$ in a Banach space $X$, we call a sequence $\left(x_{k}\right)$ in $X$ as a Weyl sequence for $T$ if $\left\|x_{k}\right\|=$ 1 and $\left\|T x_{k}\right\| \rightarrow 0$, as $k \rightarrow \infty$.

In what follows, we call the set

$$
\begin{aligned}
\sigma_{\text {ap }}(T, X) \\
\quad:=\{\alpha \in \mathbb{C}: \text { there exists a Weyl sequence for } \alpha I-T\}
\end{aligned}
$$

the approximate point spectrum of T. Moreover, the subspectrum

$$
\sigma_{\delta}(T, X):=\{\alpha \in \mathbb{C}: \alpha I-T \text { is not surjective }\}
$$

is called defect spectrum of $T$.

The two subspectra given by (3) and (4) form a (not necessarily disjoint) subdivisions

$$
\sigma(T, X)=\sigma_{\text {ap }}(T, X) \cup \sigma_{\delta}(T, X)
$$

of the spectrum. There is another subspectrum

$$
\sigma_{\text {co }}(T, X)=\{\alpha \in \mathbb{C}: \overline{R(\alpha I-T)} \neq X\}
$$

which is often called compression spectrum in the literature.

By the definitions given above, we can illustrate the subdivisions of spectrum in Table 1.

From Goldberg [16] if $T \in B(X), X$ a Banach space, then there are three possibilities for $R(T)$ :

(I) $R(T)=X$,

(II) $R(T) \neq \overline{R(T)}=X$,

(III) $\overline{R(T)} \neq X$,

and three possibilities for $T^{-1}$ :

(1) $T^{-1}$ exists and is continuous,

(2) $T^{-1}$ exists but is discontinuous,

(3) $T^{-1}$ does not exist.

If these possibilities are combined in all possible ways, nine different states are created. These are labelled by $I_{1}, I_{2}$, $I_{3}, I I_{1}, I I_{2}, I I_{3}, I I I_{1}, I I I_{2}$ and $I I I_{3}$. If $\alpha$ is a complex number such that $T_{\alpha} \in I_{1}$ or $T_{\alpha} \in I I_{1}$, then $\alpha$ is in the resolvent set $\rho(X, T)$ of $T$. The further classification gives rise to the fine spectrum of $T$. If an operator is in state $I I_{2}$, for example, then $R(T) \neq \overline{R(T)}=X$ and $T^{-1}$ exists but is discontinuous and we write $\alpha \in I I_{2} \sigma(X, T)$.

Let $\mu$ and $\gamma$ be two sequence spaces and let $A=\left(a_{n k}\right)$ be an infinite matrix of real or complex numbers $a_{n k}$, where $n, k \in \mathbb{N}=\{0,1,2, \ldots\}$. Then, we say that $A$ defines a matrix 
mapping from $\mu$ into $\gamma$ and we denote it by writing $A: \mu \rightarrow$ $\gamma$ if for every sequence $x=\left(x_{k}\right) \in \mu$ the sequence $A x=$ $\left\{(A x)_{n}\right\}$, the $A$-transform of $x$ is in $\gamma$, where

$$
(A x)_{n}=\sum_{k} a_{n k} x_{k} \quad \text { for each } n \in \mathbb{N} .
$$

By $(\mu: \gamma)$, we denote the class of all matrices $A$ such that $A: \mu \rightarrow \gamma$. Thus, $A \in(\mu: \gamma)$ if and only if the series on the right side of (7) converges for each $n \in \mathbb{N}$ and every $x \in \mu$, and we have $A x=\left\{(A x)_{n}\right\}_{n \in \mathbb{N}} \in \gamma$ for all $x \in \mu$.

Proposition 1 (see [15, Proposition 1.3, p. 28]). Spectra and subspectra of an operator $T \in B(X)$ and its adjoint $T^{*} \in B\left(X^{*}\right)$ are related by the following relations:

(a) $\sigma\left(T^{*}, X^{*}\right)=\sigma(T, X)$,

(b) $\sigma_{c}\left(T^{*}, X^{*}\right) \subseteq \sigma_{\text {ap }}(T, X)$,

(c) $\sigma_{\text {ap }}\left(T^{*}, X^{*}\right)=\sigma_{\delta}(T, X)$,

(d) $\sigma_{\delta}\left(T^{*}, X^{*}\right)=\sigma_{\text {ap }}(T, X)$,

(e) $\sigma_{p}\left(T^{*}, X^{*}\right)=\sigma_{\text {co }}(T, X)$,

(f) $\sigma_{\mathrm{co}}\left(T^{*}, X^{*}\right) \supseteq \sigma_{p}(T, X)$,

(g) $\sigma(T, X)=\sigma_{\text {ap }}(T, X) \cup \sigma_{p}\left(T^{*}, X^{*}\right)=\sigma_{p}(T, X) \cup$ $\sigma_{\text {ap }}\left(T^{*}, X^{*}\right)$.

The relations $(c)-(f)$ show that the approximate point spectrum is in a certain sense dual to defect spectrum and the point spectrum dual to the compression spectrum.

The equality $(g)$ implies, in particular, that $\sigma(T, X)=$ $\sigma_{\mathrm{ap}}(T, X)$ if $X$ is a Hilbert space and $T$ is normal. Roughly speaking, this shows that normal (in particular, self-adjoint) operators on Hilbert spaces are most similar to matrices in finite dimensional spaces (see [15]).

Lemma 2 (see [16, p. 60]). The adjoint operator $T^{*}$ of $T$ is onto if and only if $T$ has a bounded inverse.

Lemma 3 (see [16, p. 59]). T has a dense range if and only if $T^{*}$ is one to one.

Our main focus in this paper is on the triple-band matrix $A(r, s, t)$, where

$$
A(r, s, t)=\left[\begin{array}{ccccc}
r & s & t & 0 & \ldots \\
0 & r & s & t & \ldots \\
0 & 0 & r & s & \ldots \\
0 & 0 & 0 & r & \ldots \\
\vdots & \vdots & \vdots & \vdots & \ddots
\end{array}\right]
$$

We assume here and after that $s$ and $t$ are complex parameters which do not simultaneously vanish. We introduce the introduce the operator $A(r, s, t)$ from $\ell_{p}$ to itself by

$$
\begin{array}{r}
A(r, s, t) x=\left(r x_{k}+s x_{k+1}+t x_{k+2}\right)_{k=0}^{\infty}, \\
\text { where } x=\left(x_{k}\right) \in \ell_{p} .
\end{array}
$$

\section{Fine Spectra of Upper Triangular Triple-Band Matrices over the Sequence Space $\ell_{p}(0<p \leqslant 1)$}

In this section, we prove that the operator $A(r, s, t): \ell_{p} \rightarrow$ $\ell_{p}$ is a bounded linear operator and compute its norm. We essentially emphasize the fine spectrum of the operator $A(r, s, t): \ell_{p} \rightarrow \ell_{p}$ in the case $0<p \leqslant 1$.

Theorem 4. The operator $A(r, s, t): \ell_{p} \rightarrow \ell_{p}$ is a bounded linear operator and

$$
\|A(r, s, t)\|_{\left(\ell_{p}: \ell_{p}\right)}=|r|^{p}+|s|^{p}+|t|^{p} .
$$

Proof. Since the linearity of the operator $A(r, s, t)$ is trivial, so it is omitted. Let us take $e^{(2)} \in \ell_{p}$. Then $A(r, s, t) e^{(2)}=$ $(t, s, r, 0, \ldots)$ and observe that

$$
\|A(r, s, t)\|_{\left(\ell_{p}: \ell_{p}\right)} \geqslant \frac{\left\|A(r, s, t) e^{(2)}\right\|_{p}}{\left\|e^{(2)}\right\|_{p}}=|r|^{p}+|s|^{p}+|t|^{p}
$$

which gives the fact that

$$
\|A(r, s, t)\|_{\left(\ell_{p}: \ell_{p}\right)} \geqslant|r|^{p}+|s|^{p}+|t|^{p} .
$$

Let $x=\left(x_{k}\right) \in \ell_{p}$, where $0<p \leqslant 1$. Then, since $\left(t x_{k+2}\right),\left(r x_{k}\right)$, and $\left(s x_{k+1}\right) \in \ell_{p}$, it is easy to see by triangle inequality that

$$
\begin{aligned}
\|A(r, s, t) x\|_{p} & =\sum_{k=0}^{\infty}\left|r x_{k}+s x_{k+1}+t x_{k+2}\right|^{p} \\
& \leqslant \sum_{k=0}^{\infty}\left|r x_{k}\right|^{p}+\sum_{k=0}^{\infty}\left|s x_{k+1}\right|^{p}+\sum_{k=0}^{\infty}\left|t x_{k+2}\right|^{p} \\
& =|r|^{p} \sum_{k=0}^{\infty}\left|x_{k}\right|^{p}+|s|^{p} \sum_{k=0}^{\infty}\left|x_{k+1}\right|^{p}+|t|^{p} \sum_{k=0}^{\infty}\left|x_{k+2}\right|^{p} \\
& =|s|^{p}\|x\|_{p}+|r|^{p}\|x\|_{p}+|t|^{p}\|x\|_{p} \\
& =\left(|r|^{p}+|s|^{p}+|t|^{p}\right)\|x\|_{p}
\end{aligned}
$$

which leads us to the result that

$$
\|A(r, s, t)\|_{\left(\ell_{p}: \ell_{p}\right)} \leqslant|r|^{p}+|s|^{p}+|t|^{p} .
$$

Therefore, by combining the inequalities (12) and (14) we see that (10) holds which completes the proof.

If $T: \ell_{p} \rightarrow \ell_{p}$ is a bounded matrix operator with the matrix $A$, then it is known that the adjoint operator $T^{*}$ : $\ell_{p}^{*} \rightarrow \ell_{p}^{*}$ is defined by the transpose of the matrix $A$. The dual space of $\ell_{p}$ is isomorphic to $\ell_{\infty}$, where $0<p<1$.

Before giving the main theorem of this section, we should note the following remark. In this work, here and in what follows, if $z$ is a complex number, then by $\sqrt{z}$ we always mean the square root of $z$ with a nonnegative real part. If $\operatorname{Re}(\sqrt{z})=0$, then $\sqrt{z}$ represents the square root of $z$ with $\operatorname{Im}(\sqrt{z})>0$. The same results are obtained if $\sqrt{z}$ represents the other square root. 
TABLE 1: Subdivisions of spectrum of a linear operator.

\begin{tabular}{|c|c|c|c|c|}
\hline & & 1 & 2 & 3 \\
\hline & & $T_{\alpha}^{-1}$ exists and is bounded & $T_{\alpha}^{-1}$ exists and is unbounded & $T_{\alpha}^{-1}$ does not exist \\
\hline A & $R(\alpha I-T)=X$ & $\alpha \in \rho(T, X)$ & - & $\alpha \in \sigma_{p}(T, X)$ \\
\hline$\Lambda$ & 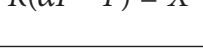 & $u=p(1, \Lambda)$ & - & $\alpha \in \sigma_{\mathrm{ap}}(T, X)$ \\
\hline & & & $\alpha \in \sigma_{c}(T, X)$ & $\alpha \in \sigma_{p}(T, X)$ \\
\hline B & $\overline{R(\alpha I-T)}=X$ & $\alpha \in \rho(T, X)$ & $\alpha \in \sigma_{\mathrm{ap}}(T, X)$ & $\alpha \in \sigma_{\mathrm{ap}}(T, X)$ \\
\hline & & & $\alpha \in \sigma_{\delta}(T, X)$ & $\alpha \in \sigma_{\delta}(T, X)$ \\
\hline & & $\alpha \in \sigma_{r}(T, X)$ & $\alpha \in \sigma_{r}(T, X)$ & $\alpha \in \sigma_{p}(T, X)$ \\
\hline $\mathrm{C}$ & $\overline{R(\alpha I-T)} \neq X$ & $\alpha \in \sigma_{\delta}(T, X)$ & $\alpha \in \sigma_{\mathrm{ap}}(T, X)$ & $\alpha \in \sigma_{\mathrm{ap}}(T, X)$ \\
\hline & & & $\alpha \in \sigma_{\delta}(T, X)$ & $\alpha \in \sigma_{\delta}(T, X)$ \\
\hline & & $\alpha \in \sigma_{\mathrm{co}}(T, X)$ & $\alpha \in \sigma_{\mathrm{co}}(T, X)$ & $\alpha \in \sigma_{\mathrm{co}}(T, X)$ \\
\hline
\end{tabular}

Theorem 5. Let $s$ be a complex number such that $\sqrt{s^{2}}=-s$ and define the set $D_{1}$ by

$$
D_{1}=\left\{\alpha \in \mathbb{C}: 2|r-\alpha| \leqslant\left|-s+\sqrt{s^{2}-4 t(r-\alpha)}\right|\right\} .
$$

Then, $\sigma_{c}\left(A(r, s, t), \ell_{p}\right) \subseteq D_{1}$.

Proof. Let $y=\left(y_{k}\right) \in \ell_{\infty}$. Then, by solving the equation $A_{\alpha}(r$, $s, t)^{*} x=y$ for $x=\left(x_{k}\right)$ in terms of $y$, we obtain

$$
\begin{aligned}
& x_{0}=\frac{y_{0}}{r-\alpha}, \\
& x_{1}=\frac{y_{1}}{r-\alpha}+\frac{-s y_{0}}{(r-\alpha)^{2}}, \\
& x_{2}=\frac{y_{2}}{r-\alpha}+\frac{-s y_{1}}{(r-\alpha)^{2}}+\frac{\left[s^{2}-t(r-\alpha)\right] y_{0}}{(r-\alpha)^{3}},
\end{aligned}
$$

and if we denote $a_{1}=1 /(r-\alpha), a_{2}=-s /(r-\alpha)^{2}$, and $a_{3}=$ $\left(s^{2}-t(r-\alpha)\right) /(r-\alpha)^{3}$, we have

$$
\begin{aligned}
& x_{0}=a_{1} y_{0}, \\
& x_{1}=a_{1} y_{1}+a_{2} y_{0}, \\
& x_{2}=a_{1} y_{2}+a_{2} y_{1}+a_{3} y_{0}, \\
& \quad \vdots \\
& x_{n}=a_{1} y_{n}+a_{2} y_{n-1}+\cdots+a_{n+1} y_{0}=\sum_{k=0}^{n} a_{n+1-k} y_{k} .
\end{aligned}
$$

Now we must find $a_{n}$. We have $y_{n}=t x_{n-2}+s x_{n-1}+(r-\alpha) x_{n}$ and if we use relation (18), we have

$$
\begin{aligned}
y_{n}= & t \sum_{k=0}^{n-2} a_{n-1-k} y_{k}+s \sum_{k=0}^{n-1} a_{n-k} y_{k}+(r-\alpha) \sum_{k=0}^{n} a_{n+1-k} y_{k} \\
= & y_{0}\left(t a_{n-1}+s a_{n}+(r-\alpha) a_{n+1}\right) \\
& +y_{1}\left(t a_{n-2}+s a_{n-1}+(r-\alpha) a_{n}\right)+\cdots+y_{n} a_{1}(r-\alpha) .
\end{aligned}
$$

This implies that

$$
\begin{gathered}
t a_{n-1}+s a_{n}+(r-\alpha) a_{n+1}=0 \\
t a_{n-2}+s a_{n-1}+(r-\alpha) a_{n}=0, \ldots, a_{1}(r-\alpha)=1 .
\end{gathered}
$$

In fact this sequence is obtained recursively by letting

$$
\begin{gathered}
a_{1}=\frac{1}{r-\alpha}, \quad a_{2}=\frac{-s}{(r-\alpha)^{2}}, \\
t a_{n-2}+s a_{n-1}+(r-\alpha) a_{n}=0, \quad \forall n \geqslant 3 .
\end{gathered}
$$

The characteristic polynomial of the recurrence relation is $(r-$ $\alpha) \lambda^{2}+s \lambda+t=0$. There are two cases.

Case 1. If $\Delta=s^{2}-4 t(r-\alpha) \neq 0$ whose roots are

$$
\lambda_{1}=\frac{-s+\sqrt{\Delta}}{2(r-\alpha)}, \quad \lambda_{2}=\frac{-s-\sqrt{\Delta}}{2(r-\alpha)},
$$

elementary calculation on recurrent sequence gives that

$$
a_{n}=\frac{\lambda_{1}^{n}-\lambda_{2}^{n}}{\sqrt{s^{2}-4 t(r-\alpha)}}, \quad \forall n \geqslant 1 .
$$

In this case $x_{k}=(1 / \sqrt{\Delta}) \sum_{k=0}^{n}\left(\lambda_{1}^{n+1-k}-\lambda_{2}^{n+1-k}\right) y_{k}$. Assume that $\left|\lambda_{1}\right|<1$. So we have

$$
\left|1+\sqrt{\frac{4 t(r-\alpha)}{s^{2}}}\right|<\left|\frac{2(r-\alpha)}{-s}\right| .
$$

Since $|1-\sqrt{z}| \leqslant|1+\sqrt{z}|$ for any $z \in \mathbb{C}$, we must have

$$
\left|1-\sqrt{\frac{4 t(r-\alpha)}{s^{2}}}\right|<\left|\frac{2(r-\alpha)}{-s}\right| .
$$

It follows that $\left|\lambda_{2}\right|<1$. Now, for $\left|\lambda_{1}\right|<1$ we can see that

$$
\left|x_{n}\right| \leqslant \frac{1}{|\sqrt{\Delta}|} \sum_{k=0}^{n}\left|\lambda_{1}^{n+1-k}\right|\left|y_{k}\right|+\sum_{k=0}^{n}\left|\lambda_{2}^{n+1-k}\right|\left|y_{k}\right|
$$

for all $n \in \mathbb{N}$. Taking limit on the inequality (26) as $n \rightarrow \infty$, we get

$$
\|x\|_{\infty} \leqslant \frac{1-\left(\left|\lambda_{2}\right|+\left|\lambda_{2}\right|\right)}{\left|\left(1-\left|\lambda_{2}\right|\left|1-\lambda_{2}\right|\right) \sqrt{\Delta}\right|}\|y\|_{\infty} .
$$


Thus for $\left|\lambda_{1}\right|<1, A_{\alpha}(r, s, t)^{*}$ is onto and by Lemma 2, $A_{\alpha}(r$, $s, t)$ has a bounded inverse. This means that

$$
\begin{aligned}
\sigma_{c}(A & \left.(r, s, t), \ell_{p}\right) \\
& \subseteq\left\{\alpha \in \mathbb{C}: 2|r-\alpha| \leqslant\left|-s+\sqrt{s^{2}-4 t(r-\alpha)}\right|\right\} \\
& =D_{1} .
\end{aligned}
$$

Case 2. If $\Delta=s^{2}-4 t(r-\alpha)=0$, a calculation on recurrent sequence gives that

$$
a_{n}=\left(\frac{2 n}{-s}\right)\left[\frac{-s}{2(r-\alpha)}\right]^{n}, \quad \forall n \geqslant 1 .
$$

Now, for $|-s|<2|r-\alpha|$ we can see that

$$
\left|x_{n}\right| \leqslant \sum_{k=0}^{n}\left|a_{n-k} y_{k}\right|
$$

for all $n \in \mathbb{N}$. Taking limit on the inequality (30) as $n \rightarrow \infty$, we obtain that

$$
\|x\|_{\infty} \leqslant\|y\|_{\infty} \sum_{k=0}^{\infty}\left|a_{k}\right| .
$$

$\sum_{k=0}^{\infty}\left|a_{k}\right|$ is convergent, since $|-s|<2|r-\alpha|$. Thus for $|-s|<$ $2|r-\alpha|, A_{\alpha}(r, s, t)^{*}$ is onto and by Lemma $2, A_{\alpha}(r, s, t)$ has a bounded inverse. This means that

$$
\sigma_{c}\left(A(r, s, t), \ell_{p}\right) \subseteq\{\alpha \in \mathbb{C}: 2|r-\alpha| \leqslant|-s|\} \subseteq D_{1} .
$$

Theorem 6. $\sigma_{p}\left(A(r, s, t)^{*}, \ell_{p}^{*}\right)=\emptyset$.

Proof. Consider $A(r, s, t)^{*} f=\alpha f$ with $f \neq \theta=(0,0,0, \ldots)$ in $\ell_{p}^{*}=\ell_{\infty}$. Then, by solving the system of linear equations

$$
\begin{gathered}
r f_{0}=\alpha f_{0}, \\
s f_{0}+r f_{1}=\alpha f_{1}, \\
t f_{0}+s f_{1}+r f_{2}=\alpha f_{2}, \\
t f_{1}+s f_{2}+r f_{3}=\alpha f_{3}, \\
\vdots \\
t f_{k-2}+s f_{k-1}+r f_{k}=\alpha f_{k},
\end{gathered}
$$

we find that $f_{0}=0$ if $\alpha \neq r$ and $f_{1}=f_{2}=\cdots=0$ if $f_{0}=0$ which contradicts $f \neq \theta$. If $f_{n_{0}}$ is the first nonzero entry of the sequence $f=\left(f_{n}\right)$ and $\alpha=r$, then we get $t f_{n_{0}-2}+s f_{n_{0}-1}+r f_{n_{0}}=$ $\alpha f_{n_{0}}$ which implies $f_{n_{0}}=0$ which contradicts the assumption $f_{n_{0}} \neq 0$. Hence, the equation $A(r, s, t)^{*} f=\alpha f$ has no solution $f \neq \theta$.
Theorem 7. $\sigma_{p}\left(A(r, s, t), \ell_{p}\right)=D_{2}$, where $D_{2}=\{\alpha \in \mathbb{C}$ : $\left.2|r-\alpha|<\left|-s+\sqrt{s^{2}-4 t(r-\alpha)}\right|\right\}$.

Proof. Let $A(r, s, t) x=\alpha x$ for $\theta \neq x \in \ell_{p}$. Then, by solving the system of linear equations

$$
\begin{gathered}
r x_{0}+s x_{1}+t x_{2}=\alpha x_{0}, \\
r x_{1}+s x_{2}+t x_{3}=\alpha x_{1}, \\
r x_{2}+s x_{3}+t x_{4}=\alpha x_{2}, \\
\vdots \\
r x_{k-2}+s x_{k-1}+t x_{k}=\alpha x_{k},
\end{gathered}
$$

and we have

$$
\begin{aligned}
x_{2}= & \frac{-s}{t} x_{1}-\frac{r-\alpha}{t} x_{0}, \\
x_{3}= & \frac{s^{2}-t(r-\alpha)}{t^{2}} x_{1}+\frac{s(r-\alpha)}{t^{2}} x_{0}, \\
& \vdots \\
x_{n}= & \frac{a_{n}(r-\alpha)^{n}}{t^{n-1}} x_{1}-\frac{a_{n-1}(r-\alpha)^{n}}{t^{n-1}} x_{0}, \quad \forall n \geqslant 2 .
\end{aligned}
$$

Assume that $\alpha \in D_{2}$. Then, we choose $x_{0}=1$ and $x_{1}=2(r-$ $\alpha) /\left(-s+\sqrt{s^{2}-4 t(r-\alpha)}\right)$. We show that $x_{n}=x_{1}^{n}$ for all $n \geqslant 2$. Since $\lambda_{1}, \lambda_{2}$ are roots of the characteristic equation $(r-\alpha) \lambda^{2}+$ $s \lambda+t=0$, we must have

$$
\lambda_{1} \lambda_{2}=\frac{t}{r-\alpha}, \quad \lambda_{1}-\lambda_{2}=\frac{\sqrt{\Delta}}{r-\alpha} .
$$

Combining the fact $x_{1}=1 / \lambda_{1}$ with relation (35), we can see that

$$
\begin{aligned}
x_{n} & =\frac{a_{n}(r-\alpha)^{n}}{t^{n-1}} x_{1}-\frac{a_{n-1}(r-\alpha)^{n}}{t^{n-1}} x_{0} \\
& =\left(\frac{r-\alpha}{t}\right)^{n-1}(r-\alpha)\left(-a_{n-1} x_{0}+a_{n} x_{1}\right) \\
& =\frac{1}{\left(\lambda_{1} \lambda_{2}\right)^{n-1}} \frac{r-\alpha}{\sqrt{\Delta}}\left(-\lambda_{1}^{n-1}+\lambda_{2}^{n-1}+\lambda_{1}^{n-1}-\lambda_{2}^{n} \lambda_{1}^{-1}\right) \\
& =\frac{1}{\lambda_{1}^{n-1} \lambda_{2}^{n-1}}\left(\frac{1}{\lambda_{1}-\lambda_{2}}\right) \lambda_{2}^{n-1}\left(\frac{\lambda_{1}-\lambda_{2}}{\lambda_{1}}\right) \\
& =\frac{1}{\lambda_{1}^{n}} \\
& =x_{1}^{n} .
\end{aligned}
$$

The same result may be obtained in case $\Delta=0$. Now $x=$ $\left(x_{k}\right) \in \ell_{p}$, since $\left|x_{1}\right|<1$. This shows that $D_{2} \subseteq \sigma_{p}(A(r$, $\left.s, t), \ell_{p}\right)$. 
Now we assume that $\alpha \notin D_{2}$, that is, $\left|\lambda_{1}\right| \leqslant 1$. We must show that $\alpha \notin \sigma_{p}\left(A(r, s, t), \ell_{p}\right)$. Therefore we obtain from the relation (35) that

$$
\frac{x_{n+1}}{x_{n}}=\left(\frac{r-\alpha}{t}\right) \frac{a_{n-1}}{a_{n-2}}\left(\frac{-x_{0}+\left(a_{n} / a_{n-1}\right) x_{1}}{-x_{0}+\left(a_{n-1} / a_{n-2}\right) x_{1}}\right) .
$$

Now we examine three cases.

Case $1\left(\left|\lambda_{2}\right|<\left|\lambda_{1}\right| \leqslant 1\right)$. In this case we have $s^{2} \neq 4 t(r-\alpha)$ and

$$
\frac{a_{n}}{a_{n-1}}=\frac{\lambda_{1}^{n+1}-\lambda_{2}^{n+1}}{\lambda_{1}^{n}-\lambda_{2}^{n}}=\frac{\lambda_{1}\left[1-\left(\lambda_{2} / \lambda_{1}\right)^{n+1}\right]}{\left[1-\left(\lambda_{2} / \lambda_{1}\right)^{n}\right]} .
$$

Then, we have

$$
\begin{aligned}
\lim _{n \rightarrow \infty}\left|\frac{a_{n}}{a_{n-1}}\right|^{p} & =\lim _{n \rightarrow \infty}\left|\frac{a_{n-1}}{a_{n-2}}\right|^{p} \\
& =\lim _{n \rightarrow \infty} \frac{\left|\lambda_{1}\right|^{p}\left|1-\left(\lambda_{2} / \lambda_{1}\right)^{n+1}\right|^{p}}{\left|1-\left(\lambda_{2} / \lambda_{1}\right)^{n}\right|^{p}}=\left|\lambda_{1}\right|^{p} .
\end{aligned}
$$

Now, if $-x_{0}+\lambda_{1} x_{1}=0$, then we have $\left(x_{n}\right)=\left(x_{0} / \lambda_{1}^{n}\right)$ which is not in $\ell_{p}$. Otherwise,

$$
\lim _{n \rightarrow \infty}\left|\frac{x_{n+1}}{x_{n}}\right|^{p}=\frac{1}{\left|\lambda_{1}\right|^{p}\left|\lambda_{2}\right|^{p}}\left|\lambda_{1}\right|^{p}=\frac{1}{\left|\lambda_{2}\right|^{p}}>1 .
$$

Case $2\left(\left|\lambda_{2}\right|=\left|\lambda_{1}\right|<1\right)$. In this case we have $s^{2}=4 t(r-\alpha)$ and using the formula

$$
a_{n}=\left(\frac{2 n}{-s}\right)\left[\frac{-s}{2(r-\alpha)}\right]^{n} \quad \forall n \geqslant 1,
$$

we obtain that

$$
\lim _{n \rightarrow \infty}\left|\frac{a_{n}}{a_{n-1}}\right|^{p}=\left|\frac{-s}{2(r-\alpha)}\right|^{p}=\left|\lambda_{1}\right|^{p}
$$

which leads to

$$
\lim _{n \rightarrow \infty}\left|\frac{x_{n+1}}{x_{n}}\right|^{p}=\frac{1}{\left|\lambda_{1}\right|^{p}\left|\lambda_{2}\right|^{p}}\left|\lambda_{1}\right|^{p}=\frac{1}{\left|\lambda_{2}\right|^{p}}>1 .
$$

Case $3\left(\left|\lambda_{2}\right|=\left|\lambda_{1}\right|=1\right)$. In this case we have $s^{2}=4 t(r-\alpha)$ and so we have $|-s /(2 t)|=1$. Assume that $\alpha \in \sigma_{p}\left(A(r, s, t), \ell_{p}\right)$. This implies that $x \in \ell_{p}$ and $x \neq \theta$. Thus we again derive (35)

$$
x_{n}=\left(\frac{-s}{2 t}\right)^{n-1}\left[-(n-1) \frac{-s}{2 t} x_{0}+n x_{1}\right] \text {. }
$$

Since $\lim _{n \rightarrow \infty} x_{n}=0$, we must have $x_{0}=x_{1}=0$. But this implies that $x=\theta$, a contradiction which means that $\alpha \notin$ $\sigma_{p}\left(A(r, s, t), \ell_{p}\right)$. Thus $\sigma_{p}\left(A(r, s, t), \ell_{p}\right) \subseteq D_{2}$. This completes the proof.

Theorem 8. $\sigma_{r}\left(A(r, s, t), \ell_{p}\right)=\emptyset$.
Proof. By Proposition 1, $\sigma_{r}\left(A(r, s, t), \ell_{p}\right)=\sigma_{p}\left(A(r, s, t)^{*}, \ell_{p}^{*}\right) \backslash$ $\sigma_{p}\left(A(r, s, t), \ell_{p}\right)$. Since by Theorem 6 ,

$$
\sigma_{p}\left(A(r, s, t)^{*}, \ell_{p}^{*}\right)=\emptyset, \quad \sigma_{r}\left(A(r, s, t), \ell_{p}\right)=\emptyset .
$$

This completes the proof.

Theorem 9. Let $s$ be a complex number such that $\sqrt{s^{2}}=-s$ and define the set $D_{1}$ by

$$
D_{1}=\left\{\alpha \in \mathbb{C}: 2|r-\alpha| \leqslant\left|-s+\sqrt{s^{2}-4 t(r-\alpha)}\right|\right\} \text {. }
$$

Then, $\sigma\left(A(r, s, t), \ell_{p}\right)=D_{1}$.

Proof. By Theorem 7, we get

$$
\begin{aligned}
\left\{\alpha \in \mathbb{C}: 2|r-\alpha|<\left|-s+\sqrt{s^{2}-4 t(r-\alpha)}\right|\right\} & \subseteq \sigma\left(A(r, s, t), \ell_{p}\right) .
\end{aligned}
$$

Since the spectrum of any bounded operator is closed, we have

$$
\begin{aligned}
\left\{\alpha \in \mathbb{C}: 2|r-\alpha| \leqslant\left|-s+\sqrt{s^{2}-4 t(r-\alpha)}\right|\right\} & \subseteq \sigma\left(A(r, s, t), \ell_{p}\right)
\end{aligned}
$$

and again from Theorems 5, 7, and 8,

$$
\begin{aligned}
\sigma\left(A(r, s, t), \ell_{p}\right) & \subseteq\{\alpha \in \mathbb{C}: 2|r-\alpha| \\
& \left.\leqslant\left|-s+\sqrt{s^{2}-4 t(r-\alpha)}\right|\right\} .
\end{aligned}
$$

Combining (49) and (50), we obtain that $\sigma\left(A(r, s, t), \ell_{p}\right)=$ $D_{1}$, where $D_{1}$ is defined by (47).

Theorem 10. $\sigma_{c}\left(A(r, s, t), \ell_{p}\right)=D_{3}$, where $D_{3}=\{\alpha \in \mathbb{C}$ : $\left.2|r-\alpha|=\left|-s+\sqrt{s^{2}-4 t(r-\alpha)}\right|\right\}$.

Proof. Because the parts $\sigma_{c}\left(A(r, s, t), \ell_{p}\right), \sigma_{r}\left(A(r, s, t), \ell_{p}\right)$, and $\sigma_{p}\left(A(r, s, t), \ell_{p}\right)$ are pairwise disjoint sets and the union of these sets is $\sigma\left(A(r, s, t), \ell_{p}\right)$, the proof immediately follows from Theorems 7, 8, and 9 .

Theorem 11. If $\alpha \in D_{2}, \alpha \in \sigma\left(A(r, s, t), \ell_{p}\right) I_{3}$.

Proof. From Theorem 7, $\alpha \in \sigma_{p}\left(A(r, s, t), \ell_{p}\right)$. Thus, $(A(r$, $s, t)-\alpha I)^{-1}$ does not exist. By Theorem $6 A(r, s, t)^{*}-\alpha I$ is one to one, so $A(r, s, t)-\alpha I$ has a dense range in $\ell_{p}$ by Lemma 3.

Theorem 12. The following statements hold:

(i) $\sigma_{\text {ap }}\left(A(r, s, t), \ell_{p}\right)=D_{1}$,

(ii) $\sigma_{\delta}\left(A(r, s, t), \ell_{p}\right)=D_{3}$,

(iii) $\sigma_{\text {co }}\left(A(r, s, t), \ell_{p}\right)=\emptyset$. 
Proof. (i) Since from Table 1,

$$
\sigma_{\mathrm{ap}}\left(A(r, s, t), \ell_{p}\right)=\sigma\left(A(r, s, t), \ell_{p}\right) \backslash \sigma\left(A(r, s, t), \ell_{p}\right) I I I_{1}
$$

we have by Theorem 8

$$
\sigma\left(A(r, s, t), \ell_{p}\right) I I I_{1}=\sigma\left(A(r, s, t), \ell_{p}\right) I I I_{2}=\emptyset .
$$

Hence

$$
\sigma_{\text {ap }}\left(A(r, s, t), \ell_{p}\right)=D_{1}
$$

(ii) Since the following equality

$$
\sigma_{\delta}\left(A(r, s, t), \ell_{p}\right)=\sigma\left(A(r, s, t), \ell_{p}\right) \backslash \sigma\left(A(r, s, t), \ell_{p}\right) I_{3}
$$

holds from Table 1, we derive by Theorems 8 and 11 that $\sigma_{\delta}\left(A(r, s, t), \ell_{p}\right)=D_{2}$.

(iii) From Table 1, we have

$$
\begin{aligned}
\sigma_{\mathrm{co}}\left(A(r, s, t), \ell_{p}\right) \\
=\sigma\left(A(r, s, t), \ell_{p}\right) I I I_{1} \cup \sigma\left(A(r, s, t), \ell_{p}\right) I I I_{2} \\
\cup \sigma\left(A(r, s, t), c_{0}\right) I I I_{3} .
\end{aligned}
$$

By Theorem 6 it is immediate that $\sigma_{\mathrm{co}}\left(A(r, s, t), \ell_{p}\right)=\emptyset$.

\section{Fine Spectra of Upper Triangular Triple-Band Matrices over the Sequence Space $\ell_{p}(1<p<\infty)$}

In the present section, we determine the fine spectrum of the operator $A(r, s, t): \ell_{p} \rightarrow \ell_{p}$ in case $1 \leqslant p<\infty$. We quote some lemmas which are needed in proving the theorems given in Section 4 .

Lemma 13 (see $[17$, p. 253, Theorem 34.16]). The matrix $A=$ $\left(a_{n k}\right)$ gives rise to a bounded linear operator $T \in B\left(\ell_{1}\right)$ from $\ell_{1}$ to itself if and only if the supremum of $\ell_{1}$ norms of the columns of $A$ is bounded.

Lemma 14 (see [17, p. 245, Theorem 34.3]). The matrix $A=$ $\left(a_{n k}\right)$ gives rise to a bounded linear operator $T \in B\left(\ell_{\infty}\right)$ from $\ell_{\infty}$ to itself if and only if the supremum of $\ell_{1}$ norms of the rows of $A$ is bounded.

Lemma 15 (see [17, p. 254, Theorem 34.18]). Let $1<p<\infty$ and $A \in\left(\ell_{\infty}: \ell_{\infty}\right) \cap\left(\ell_{1}: \ell_{1}\right)$. Then, $A \in\left(\ell_{p}: \ell_{p}\right)$.

Theorem 16. The operator $A(r, s, t): \ell_{p} \rightarrow \ell_{p}$ is a bounded linear operator and

$$
\left(|r|^{p}+|s|^{p}+|t|^{p}\right)^{1 / p} \leqslant\|A(r, s, t)\|_{\left(\ell_{p}: \ell_{p}\right)} \leqslant|r|+|s|+|t| .
$$

Proof. Since the linearity of the operator $A(r, s, t)$ is not hard, we omit the details. Now we prove that (56) holds for the operator $A(r, s, t)$ on the space $\ell_{p}$. It is trivial that $A(r, s$, t) $e^{(2)}=(t, s, r, 0, \ldots)$ for $e^{(2)} \in \ell_{p}$. Therefore, we have

$$
\|A(r, s, t)\|_{\left(\ell_{p}: \ell_{p}\right)} \geqslant \frac{\left\|A(r, s, t) e^{(2)}\right\|_{p}}{\left\|e^{(2)}\right\|_{p}}=\left(|r|^{p}+|s|^{p}+|t|^{p}\right)^{1 / p}
$$

which implies that

$$
\|A(r, s, t)\|_{\left(\ell_{p}: \ell_{p}\right)} \geqslant\left(|r|^{p}+|s|^{p}+|t|^{p}\right)^{1 / p} .
$$

Let $x=\left(x_{k}\right) \in \ell_{p}$, where $1<p<\infty$. Then, since $\left(t x_{k+2}\right)$, $\left(r x_{k}\right)$, and $\left(s x_{k+1}\right) \in \ell_{p}$, it is easy to see by Minkowski's inequality that

$$
\begin{aligned}
\|A(r, s, t) x\|_{p} & \left(\sum_{k=0}^{\infty}\left|r x_{k}+s x_{k+1}+t x_{k+2}\right|^{p}\right)^{1 / p} \\
\leqslant & \left(\sum_{k=0}^{\infty}\left|r x_{k}\right|^{p}\right)^{1 / p}+\left(\sum_{k=0}^{\infty}\left|s x_{k+1}\right|^{p}\right)^{1 / p} \\
& +\left(\sum_{k=0}^{\infty}\left|t x_{k+2}\right|^{p}\right)^{1 / p} \\
= & |r|\left(\sum_{k=0}^{\infty}\left|x_{k}\right|^{p}\right)^{1 / p}+|s|\left(\sum_{k=0}^{\infty}\left|x_{k+1}\right|^{p}\right)^{1 / p} \\
& +|t|\left(\sum_{k=0}^{\infty}\left|x_{k+2}\right|^{p}\right)^{1 / p} \\
= & |s|\|x\|_{p}+|r|\|x\|_{p}+|t|\|x\|_{p} \\
= & (|r|+|s|+|t|)\|x\|_{p}
\end{aligned}
$$

which leads us to the result that

$$
\left(|r|^{p}+|s|^{p}+|t|^{p}\right)^{1 / p} \leqslant\|A(r, s, t)\|_{\left(\ell_{p}: \ell_{p}\right)} \leqslant|r|+|s|+|t| .
$$

Therefore, by combining the inequalities in (58) and (59) we have (56), as desired.

If $T: \ell_{p} \rightarrow \ell_{p}$ is a bounded matrix operator with the matrix $A$, then it is known that the adjoint operator $T^{*}$ : $\ell_{p}^{*} \rightarrow \ell_{p}^{*}$ is defined by the transpose of the matrix $A$. The dual space of $\ell_{p}$ is isomorphic to $\ell_{q}$, where $1<p<\infty$.

Theorem 17. Let $s$ be a complex number such that $\sqrt{s^{2}}=-s$ and define the set $D_{1}$ by

$$
D_{1}=\left\{\alpha \in \mathbb{C}: 2|r-\alpha| \leqslant\left|-s+\sqrt{s^{2}-4 t(r-\alpha)}\right|\right\} .
$$

Then, $\sigma_{c}\left(A(r, s, t), \ell_{p}\right) \subseteq D_{1}$. 
Proof. We will show that $A_{\alpha}(r, s, t)^{*}$ is onto, for $2|r-\alpha|>$ $\left|-s+\sqrt{s^{2}-4 t(r-\alpha)}\right|$. Thus, for every $y \in \ell_{q}$, we find $x \in$ $\ell_{q} \cdot A_{\alpha}(r, s, t)^{*}$ is a triangle so it has an inverse. Also equation $A_{\alpha}(r, s, t)^{*} x=y$ gives $\left[A_{\alpha}(r, s, t)^{*}\right]^{-1} y=x$. It is sufficient to show that $\left[A_{\alpha}(r, s, t)^{*}\right]^{-1} \in\left(\ell_{q}: \ell_{q}\right)$. We calculate that $A=\left(a_{n k}\right)=\left[A_{\alpha}(r, s, t)^{*}\right]^{-1}$ as follows:

$$
A=\left(a_{n k}\right)=\left[\begin{array}{cccc}
a_{1} & 0 & 0 & \ldots \\
a_{2} & a_{1} & 0 & \ldots \\
a_{3} & a_{2} & a_{1} & \ldots \\
\vdots & \vdots & \vdots & \ddots
\end{array}\right]
$$

where

$$
\begin{aligned}
& a_{1}=\frac{1}{r-\alpha}, \\
& a_{2}=\frac{-s}{(r-\alpha)^{2}}, \\
& a_{3}=\frac{s^{2}-t(r-\alpha)}{(r-\alpha)^{3}},
\end{aligned}
$$

It is known that from Theorem 5

$$
\begin{aligned}
& a_{n}=\frac{\lambda_{1}^{n}-\lambda_{2}^{n}}{\sqrt{s^{2}-4 t(r-\alpha)}}, \quad \forall n \geqslant 1, \\
& \quad \text { where } \lambda_{1}=\frac{-s+\sqrt{\Delta}}{r-\alpha}, \quad \lambda_{2}=\frac{-s-\sqrt{\Delta}}{r-\alpha} .
\end{aligned}
$$

Now, we show that $\left[A_{\alpha}(r, s, t)^{*}\right]^{-1} \in\left(\ell_{1}: \ell_{1}\right)$, for $\left|\lambda_{1}\right|<1$. By Theorem 5, we know that if $\left|\lambda_{1}\right|<1,\left|\lambda_{2}\right|<1$. We assume that $s^{2}-4 t(r-\alpha) \neq 0$ and $\left|\lambda_{1}\right|<1$,

$$
\begin{aligned}
\left\|\left[A_{\alpha}(r, s, t)^{*}\right]^{-1}\right\|_{\left(\ell_{1}: \ell_{1}\right)} & =\sup _{n} \sum_{k=n}^{\infty}\left|a_{k}\right|=\sum_{k=1}^{\infty}\left|a_{k}\right| \\
& \leqslant \frac{1}{|\Delta|}\left(\sum_{k=1}^{\infty}\left|\lambda_{1}\right|^{k}+\sum_{k=1}^{\infty}\left|\lambda_{2}\right|^{k}\right)<\infty,
\end{aligned}
$$

since $\left|\lambda_{1}\right|<1$ and $\left|\lambda_{2}\right|<1$. This shows that $\left(A_{\alpha}(r, s, t)^{*}\right]^{-1} \in$ $\left(\ell_{1}: \ell_{1}\right)$. Similarly we can show that $\left[A_{\alpha}(r, s, t)^{*}\right]^{-1} \in\left(\ell_{\infty}\right.$ : $\left.\ell_{\infty}\right)$.

Now assume that $s^{2}-4 t(r-\alpha)=0$. Then,

$$
a_{n}=\left(\frac{2 n}{-s}\right)\left[\frac{-s}{2(r-\alpha)}\right]^{n}
$$

and simple calculation gives that $\left(a_{n}\right) \in \ell_{q}$ if and only if $|-s|<$ $2|r-\alpha|$

$$
\begin{aligned}
& {\left[(A(r, s, t)-\alpha I)^{*}\right]^{-1} \in\left(\ell_{q}: \ell_{q}\right)} \\
& \quad \text { for } \alpha \in \mathbb{C} \text { with } 2|r-\alpha|>\left|-s+\sqrt{s^{2}-4 t(r-\alpha)}\right| .
\end{aligned}
$$

Hence, $A_{\alpha}(r, s, t)^{*}$ is onto. By Lemma $2, A_{\alpha}(r, s, t)$ has a bounded inverse. This means that $\sigma_{c}\left(A(r, s, t), \ell_{p}\right) \subseteq D_{1}$, where $D_{1}$ is defined by (61).

Theorem 18. $\sigma_{p}\left(A(r, s, t)^{*}, \ell_{p}^{*}\right)=\emptyset$.

Proof. Let $A(r, s, t)^{*} f=\alpha f$ with $f \neq \theta=(0,0,0, \ldots)$ in $\ell_{p}^{*}=$ $\ell_{q}$. Then, by solving the system of linear equations

$$
\begin{gathered}
r f_{0}=\alpha f_{0}, \\
s f_{0}+r f_{1}=\alpha f_{1}, \\
t f_{0}+s f_{1}+r f_{2}=\alpha f_{2}, \\
t f_{1}+s f_{2}+r f_{3}=\alpha f_{3}, \\
\vdots \\
t f_{k-2}+s f_{k-1}+r f_{k}=\alpha f_{k},
\end{gathered}
$$

we find that $f_{0}=0$ if $\alpha \neq r$ and $f_{1}=f_{2}=\cdots=0$ if $f_{0}=0$ which contradicts $f \neq \theta$. If $f_{n_{0}}$ is the first nonzero entry of the sequence $f=\left(f_{n}\right)$ and $\alpha=r$, then we get $t f_{n_{0}-2}+s f_{n_{0}-1}+$ $r f_{n_{0}}=\alpha f_{n_{0}}$ which implies $f_{n_{0}}=0$ which contradicts the assumption $f_{n_{0}} \neq 0$. Hence, the equation $A(r, s, t)^{*} f=\alpha f$ has no solution $f \neq \theta$.

In the case $1<p<\infty$, since the proof of the theorems, in Section 4, determining the spectrum and fine spectrum of the matrix operator $A(r, s, t)$ on the sequence space $\ell_{p}$ is similar to the case $0<p \leqslant 1$; to avoid the repetition of similar statements, we give the results by the following theorem without proof.

Theorem 19. The following statements hold:
(i) $\sigma\left(A(r, s, t), \ell_{p}\right)=D_{1}$,
(ii) $\sigma_{r}\left(A(r, s, t), \ell_{p}\right)=\emptyset$,
(iii) $\sigma_{p}\left(A(r, s, t), \ell_{p}\right)=D_{2}$,
(iv) $\sigma_{c}\left(A(r, s, t), \ell_{p}\right)=D_{3}$,
(v) $\sigma_{\text {ap }}\left(A(r, s, t), \ell_{p}\right)=D_{1}$,
(vi) $\sigma_{\mathrm{co}}\left(A(r, s, t), \ell_{p}\right)=\emptyset$,
(vii) $\sigma_{\delta}\left(A(r, s, t), \ell_{p}\right)=D_{3}$.

\section{Some Applications}

In this section, we give two theorems related to Toeplitz matrix.

Theorem 20. Let $P$ be a polynomial that corresponds to the $n$ tuple $a$ and let $z_{1}, z_{2}, z_{3}, \ldots, z_{n-1}$ also be the roots of $P$. Define $T$ as a Toeplitz matrix associated with $P$, that is,

$$
T=\left[\begin{array}{ccccccccc}
a_{0} & a_{1} & a_{2} & \ldots & a_{n} & 0 & 0 & 0 & \ldots \\
0 & a_{0} & a_{1} & a_{2} & \ldots & a_{n} & 0 & 0 & \ldots \\
0 & 0 & a_{0} & a_{1} & a_{2} & \ldots & a_{n} & 0 & \ldots \\
\vdots & \vdots & \ddots & \ddots & \ddots & \ddots & \ddots & \ddots & \ddots
\end{array}\right] .
$$


The resolvent operator $T$ over $\ell_{p}$ with $1<p<\infty$, where the domain of the resolvent operator is the whole space $\ell_{p}$, exists if and if only all the roots of the polynomial are outside the unit disc $\{z \in \mathbb{C}:|z| \leqslant 1\}$. That is $T^{-1} \in\left(\ell_{p}: \ell_{p}\right)$ if and if only $\left|z_{i}\right|>1,1 \leqslant i \leqslant n-1$. In this case the resolvent operator is represented by

$$
T^{-1}=\frac{1}{a_{n-1}} A^{-1}\left(-z_{1}, 1\right) A^{-1}\left(-z_{2}, 1\right) \cdots A^{-1}\left(-z_{n-1}, 1\right),
$$

where

$$
A^{-1}\left(-z_{i}, 1\right)=-\left[\begin{array}{cccccc}
1 / z_{i} & 1 / z_{i}^{2} & 1 / z_{i}^{3} & 1 / z_{i}^{4} & 1 / z_{i}^{5} & \cdots \\
0 & 1 / z_{i} & 1 / z_{i}^{2} & 1 / z_{i}^{3} & 1 / z_{i}^{4} & \cdots \\
0 & 0 & 1 / z_{i} & 1 / z_{i}^{2} & 1 / z_{i}^{3} & \cdots \\
0 & 0 & 0 & 1 / z_{i} & 1 / z_{i}^{2} & \ldots \\
0 & 0 & 0 & 0 & 1 / z_{i} & \cdots \\
\vdots & \vdots & \vdots & \vdots & \vdots & \ddots
\end{array}\right] .
$$

Proof. Suppose all the roots of the polynomial $P(z)=a_{0}+$ $a_{1} z+\cdots+a_{n-1} z^{n-1}=a_{n}\left(z-z_{1}\right)\left(z-z_{2}\right) \cdots\left(z-z_{n-1}\right)$ are outside the unit disc. The Toeplitz matrix associated with $P$ can be written as the product

$$
T=a_{n} A\left(-z_{1}, 1\right) A\left(-z_{2}, 1\right) \cdots A\left(-z_{n-1}, 1\right)
$$

Since multiplication of upper triangular Toeplitz matrices is commutative, we can see that

$$
T^{-1}=\frac{1}{a_{n-1}} A^{-1}\left(-z_{1}, 1\right) A^{-1}\left(-z_{2}, 1\right) \cdots A^{-1}\left(-z_{n-1}, 1\right)
$$

is left inverse of $T$. Since all roots are outside the unit disc, then

$$
\left\|T^{-1}\left(-z_{i}, 1\right)\right\|_{\left(\ell_{\infty}: \ell_{\infty}\right)}=\sup _{n} \sum_{k=n}^{\infty} \frac{1}{\left|z_{i}\right|^{k+1-n}}=\sum_{k=1}^{\infty} \frac{1}{\left|z_{i}\right|^{k}}<\infty .
$$

Therefore each $T^{-1}\left(-z_{i}, 1\right) \in\left(\ell_{\infty}: \ell_{\infty}\right)$, for $1 \leqslant i \leqslant n-1$. Similarly we can say that $T^{-1}\left(-z_{i}, 1\right) \in\left(\ell_{1}: \ell_{1}\right)$. So we have $T^{-1}\left(-z_{i}, 1\right) \in\left(\ell_{p}: \ell_{p}\right)$.

Theorem 21. The resolvent operator of $A(r, s, t)$ over $\ell_{p}$ with $1<p<\infty$, where the domain of the resolvent operator is the space $\ell_{p}$, exists if and only if $2|r|>\left|-s+\sqrt{s^{2}-4 t r}\right|$. In this case, the resolvent operator is represented by the infinite banded Toeplitz matrix

$$
\begin{aligned}
& E(r, s, t) \\
& \quad=\frac{1}{t}\left[\begin{array}{ccccc}
u_{1} & u_{1}^{2} & u_{1}^{3} & u_{1}^{4} & \ldots \\
0 & u_{1} & u_{1}^{2} & u_{1}^{3} & \ldots \\
0 & 0 & u_{1} & u_{1}^{2} & \ldots \\
0 & 0 & 0 & u_{1} & \ldots \\
\vdots & \vdots & \vdots & \vdots & \ddots
\end{array}\right]\left[\begin{array}{ccccc}
u_{2} & u_{2}^{2} & u_{2}^{3} & u_{2}^{4} & \ldots \\
0 & u_{2} & u_{2}^{2} & u_{2}^{3} & \ldots \\
0 & 0 & u_{2} & u_{2}^{2} & \ldots \\
0 & 0 & 0 & u_{2} & \ldots \\
\vdots & \vdots & \vdots & \vdots & \ddots
\end{array}\right] \text {, } \\
& \text { where } u_{1}=\frac{-s+\sqrt{s^{2}-4 t r}}{2 r}, \quad u_{2}=\frac{-s-\sqrt{s^{2}-4 t r}}{2 r} .
\end{aligned}
$$

Proof. By Theorem 20, we can see that $E(r, s, t)$ is inverse of the matrix of $A(r, s, t)$. But this is not enough to say it is resolvent operator. By Lemmas 13, 14, and 15, $E(r, s, t) \in$ $\left(\ell_{p}: \ell_{p}\right)$, when $2|r|>\left|-s+\sqrt{s^{2}-4 t r}\right|$. That is for $2|r|>$ $\left|-s+\sqrt{s^{2}-4 t r}\right|, E(r, s, t)$ is a resolvent operator.

\section{Acknowledgments}

The authors would like to thank the referee for pointing out some mistakes and misprints in the earlier version of this paper. They would like to express their pleasure to Ms. Medine Yeşilkayagil, Department of Mathematics, Uşak University, 1 Eylül Campus, Uşak, Turkey, for many helpful suggestions and interesting comments on the revised form of the paper.

\section{References}

[1] M. Gonzàlez, "The fine spectrum of the Cesàro operator in $\ell_{p}$ $(1<p<\infty)$," Archiv der Mathematik, vol. 44, no. 4, pp. 355358,1985

[2] P. J. Cartlidge, Weighted mean matrices as operators on $\ell^{p}$ [Ph.D. dissertation], Indiana University, 1978.

[3] J. I. Okutoyi, "On the spectrum of $C_{1}$ as an operator on $b v_{0}$ ", Australian Mathematical Society A, vol. 48, no. 1, pp. 79-86, 1990.

[4] J. I. Okutoyi, "On the spectrum of $C_{1}$ as an operator on $b v$," Communications A, vol. 41, no. 1-2, pp. 197-207, 1992.

[5] M. Yildırım, "On the spectrum of the Rhaly operators on $\ell_{p}$," Indian Journal of Pure and Applied Mathematics, vol. 32, no. 2, pp. 191-198, 2001.

[6] B. Altay and F. Başar, "On the fine spectrum of the difference operator $\Delta$ on $c_{0}$ and c," Information Sciences, vol. 168, no. 1-4, pp. 217-224, 2004.

[7] B. Altay and F. Başar, "On the fine spectrum of the generalized difference operator $B(r, s)$ over the sequence spaces $c_{0}$ and c," International Journal of Mathematics and Mathematical Sciences, vol. 2005, no. 18, pp. 3005-3013, 2005.

[8] A. M. Akhmedov and F. Başar, "On the fine spectra of the difference operator $\Delta$ over the sequence space $\ell_{p},(1 \leq p<\infty)$," Demonstratio Mathematica, vol. 39, no. 3, pp. 585-595, 2006. 
[9] A. M. Akhmedov and F. Başar, "The fine spectra of the difference operator $\Delta$ over the sequence space $b v_{p},(1 \leq p<\infty)$," Acta Mathematica Sinica, vol. 23, no. 10, pp. 1757-1768, 2007.

[10] F. Başar and B. Altay, "On the space of sequences of p-bounded variation and related matrix mappings," Ukrainian Mathematical Journal, vol. 55, no. 1, pp. 136-147, 2003.

[11] H. Furkan, H. Bilgiç, and F. Başar, "On the fine spectrum of the operator $B(r, s, t)$ over the sequence spaces $\ell_{p}$ and $b v_{p},(1<p<$ $\infty)$," Computers \& Mathematics with Applications, vol. 60, no. 7, pp. 2141-2152, 2010.

[12] V. Karakaya and M. Altun, "Fine spectra of upper triangular double-band matrices," Journal of Computational and Applied Mathematics, vol. 234, no. 5, pp. 1387-1394, 2010.

[13] A. Karaisa, "Fine spectra of upper triangular double-band matrices over the sequence space $\ell_{p},(1<p<\infty)$," Discrete Dynamics in Nature and Society, vol. 2012, Article ID 381069, 19 pages, 2012.

[14] E. Kreyszig, Introductory Functional Analysis with Applications, John Wiley \& Sons, New York, NY, USA, 1978.

[15] J. Appell, E. Pascale, and A. Vignoli, Nonlinear Spectral Theory, vol. 10 of de Gruyter Series in Nonlinear Analysis and Applications, Walter de Gruyter, Berlin, Germany, 2004.

[16] S. Goldberg, Unbounded Linear Operators, Dover Publications, New York, NY, USA, 1985.

[17] B. Choudhary and S. Nanda, Functional Analysis with Applications, John Wiley \& Sons, New York, NY, USA, 1989. 


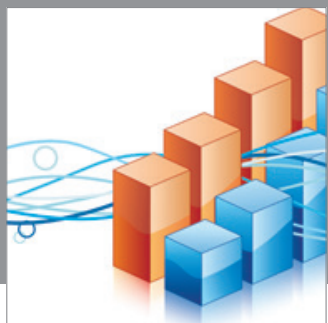

Advances in

Operations Research

mansans

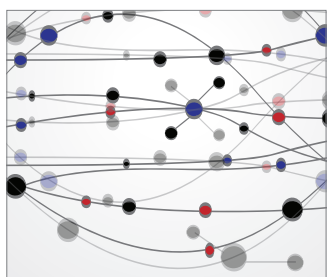

The Scientific World Journal
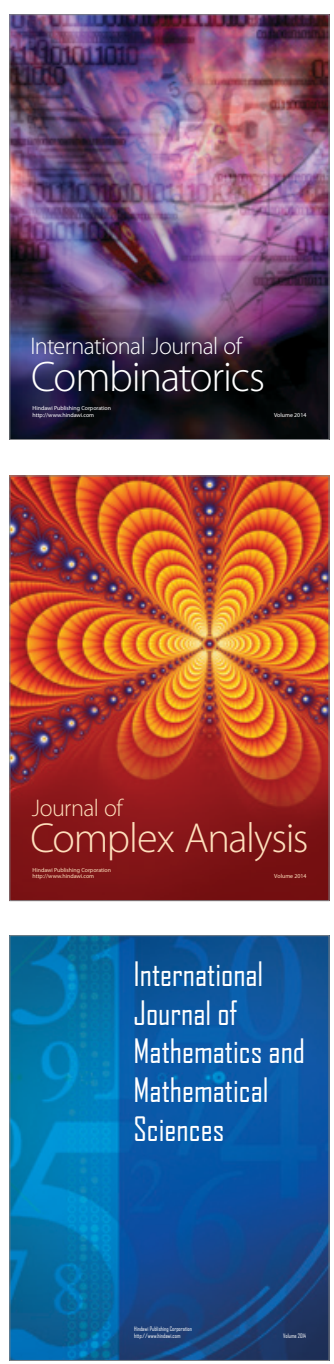
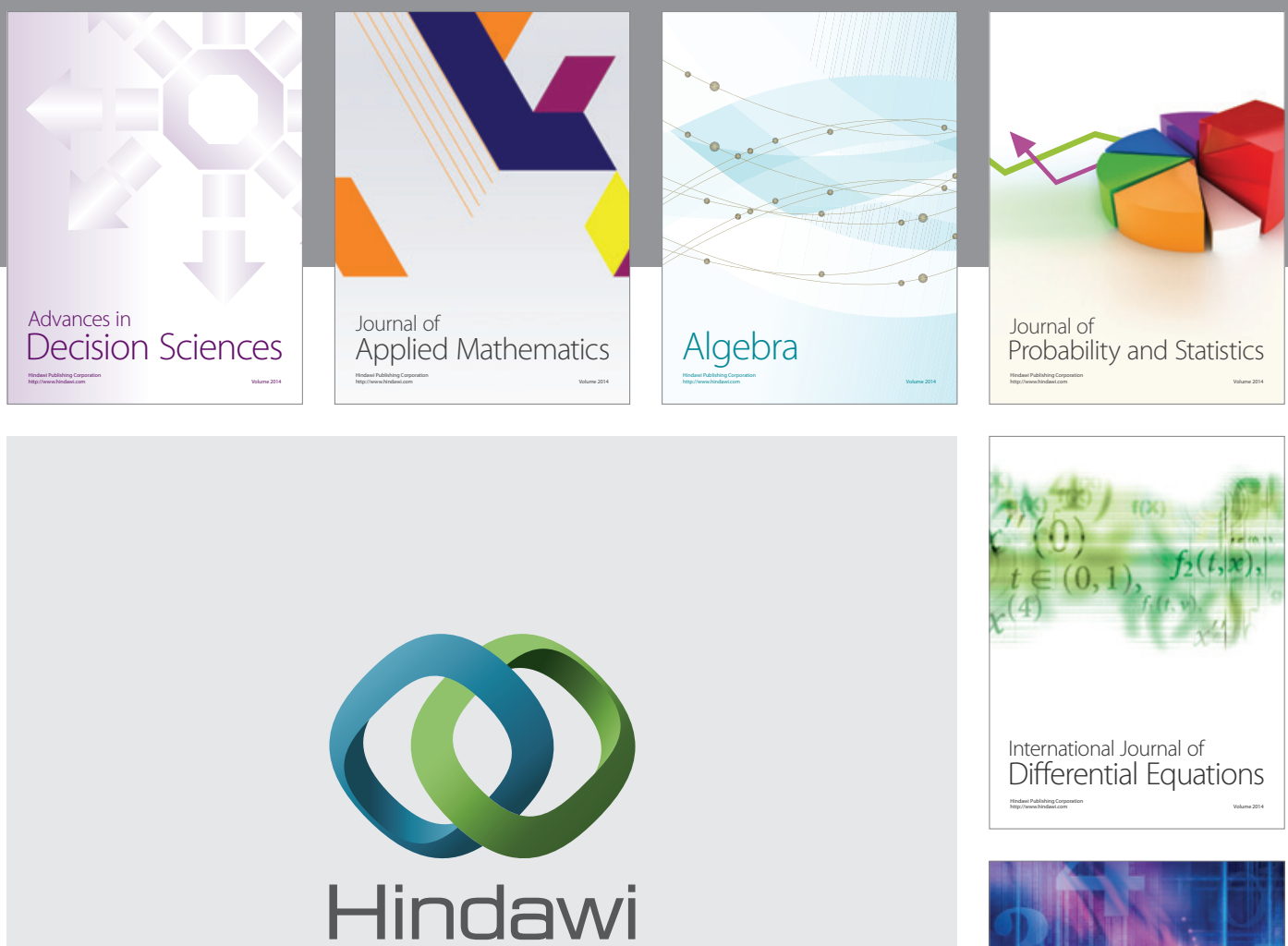

Submit your manuscripts at http://www.hindawi.com
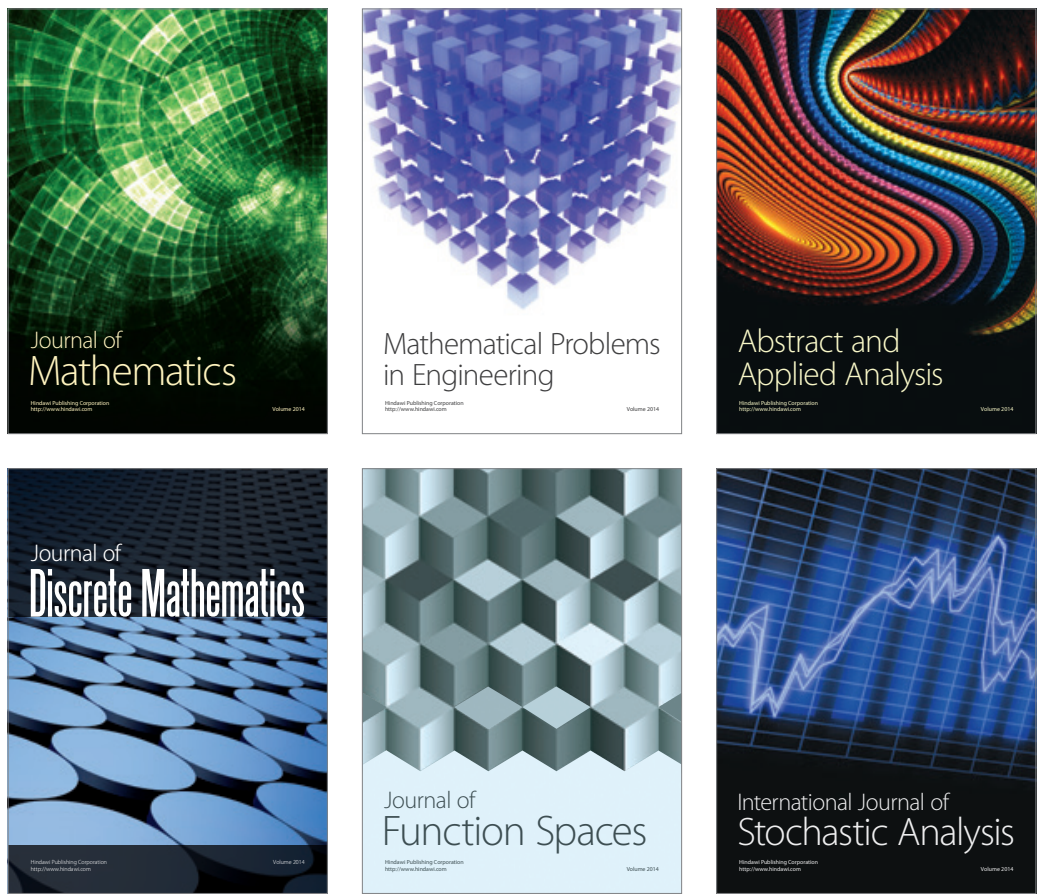

Journal of

Function Spaces

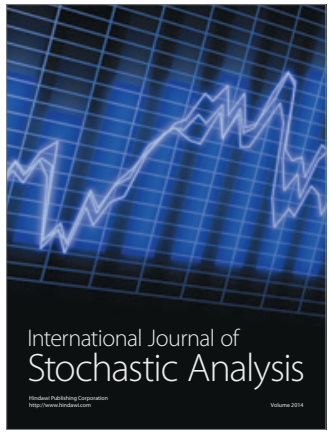

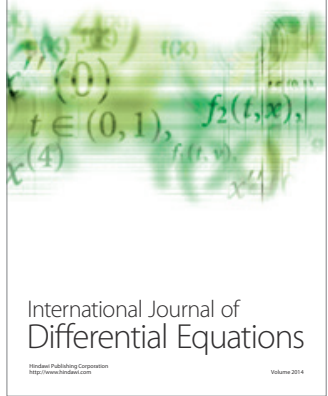
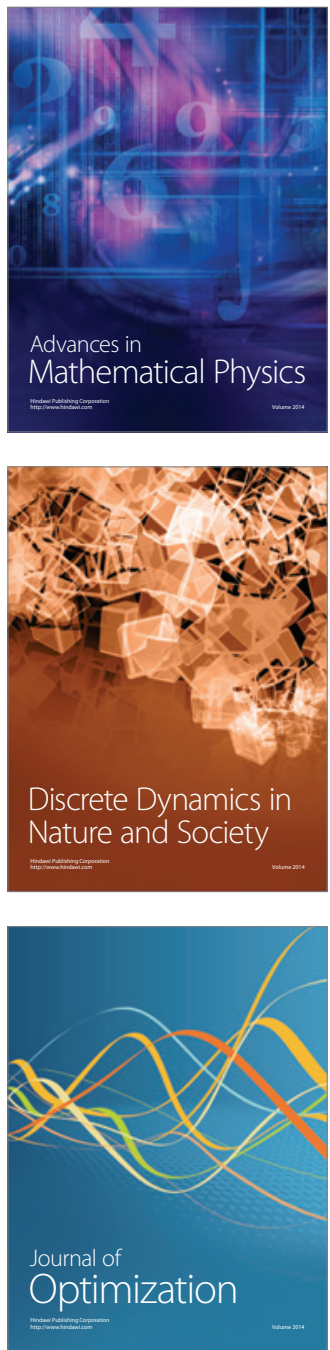